\title{
Gender-role alternation in the simultaneously hermaphroditic freshwater snail Physa acuta: not with the same partner
}

\author{
Benoît Facon • Virginie Ravigné • Jérôme Goudet
}

Received: 3 April 2007 /Revised: 2 August 2007 / Accepted: 31 August 2007 / Published online: 26 September 2007

(C) Springer-Verlag 2007

\begin{abstract}
In simultaneous hermaphrodites, gender conflicts that arise from two potential mates sharing the same gender preference may be solved through conditional reciprocity (or gamete trading). Conditional reciprocity had initially been considered widespread, but recent studies suggest that its real occurrence may have been overestimated, possibly because most mating observations have been performed on isolated pairs of individuals. Some resulting patterns (e.g., non-random alternation of sexual roles) were indeed compatible with conditional reciprocity but could also have stemmed from the two partners independently executing their own mating strategy and being experimentally enforced to do so with the same partner. Non-random alternation of
\end{abstract}

Communicated by P. Moore

B. Facon · J. Goudet

Department of Ecology and Evolution, University of Lausanne,

Biophore,

1015 Dorigny, Switzerland

V. Ravigné

UMR BGPI, Centre de coopération Internationale en Recherche

Agronomique pour le Développement,

Campus International de Baillarguet,

TA A $54 / \mathrm{K}$,

34398 Montpellier Cedex 05, France

Present address:

B. Facon $(\square)$

Institut des Sciences de l'Evolution, cc65,

Université Montpellier 2,

Place Eugène Bataillon,

34095 Montpellier Cedex 5, France

e-mail: Benoit.Facon@isem.univ-montp2.fr gender roles was recently documented in the simultaneously hermaphroditic freshwater snail Physa acuta. To distinguish between conditional and unconditional gender alternations, we observed copulations of individually marked snails reared at three contrasted densities. We showed that density affected the overall frequency of copulations during the first 2 days of the experiment with high-density boxes showing more copulations than low density boxes, but it did not affect gender alternation patterns. A change in gender role was observed more often than expected by chance over two successive copulations by the same individual, confirming previous studies. However, gender switches did not preferentially occur with the same partner. We conclude that gender alternation is not due to conditional reciprocity in P. acuta. It may rather stem from each individual having a preference for gender alternation. We finally discuss the mechanisms and the potential extent of this unconditional reciprocity.

Keywords Mating behaviour.

Conditional reciprocity $\cdot$ Hermaphroditic

freshwater snail $\cdot$ Sex role alternation

\section{Introduction}

In simultaneous hermaphrodites, gender preference may be a source of conflict among individuals (Charnov 1979; Michiels 1998). In many species, one gender role may be less costly than the other in terms of gamete production (e.g., the male role; Locher and Baur 2000), parental care (e.g., the female role; Leonard 2006; reviewed in Bishop and Pemberton 2006), or resource allocation (e.g., nuptial gifts; Michiels 1998). In addition, 
one gender role may have a lower variance in reproductive success. This is notably true for the female role in internally fertilizing species with sperm competition and cryptic sperm selection, and for the male role in many externally fertilizing species (Leonard 2006). As a consequence, one gender role may be preferred over the other if it is less costly (Bateman's principle, Bateman 1948; Charnov 1979) or less variable in terms of reproductive success (Gillespie's principle, Leonard and Lukowiak 1991; Leonard 1999). Such a species-wide preference for one gender role would provide frequent opportunities for conflicts between mating partners, that Leonard (1990) called "the hermaphrodite's dilemma". Under such a situation, selection would favor cheaters that only allocate resources to the preferred gender possibly leading to gonochorism and the disappearance of hermaphroditism. One possible mechanism by which this "tragedy of the commons" may be avoided is conditional reciprocity where each individual cooperates as far as the partner acts cooperatively, and retaliates against cheaters (Axelrod and Hamilton 1981; Leonard 1990; Dall and Wedell 2005). In simultaneous hermaphrodites, conditional reciprocity could take the form of gamete (either sperm or egg) trading (Leonard and Lukowiak 1984; Dall and Wedell 2005), where individuals exchange opportunities to fertilize or to be fertilized and stop the mating session in case of partner defection. Conditional reciprocity could thus take the form of insemination of balanced amounts of sperm in species with crossed mating or gender alternation over successive copulations in species with unilateral mating. Conditional reciprocity in the form of sperm trading should be observed in species presenting dense mating aggregations, a sperm storage organ and/or sperm digestion (Michiels and Bakovski 2000; Anthes et al. 2006a).

Following its original description (in the fish Hypoplectrus nigricans, Fischer 1980), conditional reciprocity over the preferred gender role has been suggested to be a widespread pattern that occurs in numerous species (e.g., Leonard 1990). These observations include non-random alternation of gender roles within mating pairs (e.g., Van Duivenboden and Ter Maat 1985), balanced durations of copulations in both gender roles (e.g., Michiels et al. 2003), and correlated amounts of exchanged gametes (as in the planarian flatworms Dugesia gonocephala Vreys and Michiels 1998 and D. polychroa, Michiels and Streng 1998 and the land snail Succinea putris, Jordaens et al. 2005). However, it has recently been pointed out that the presence of reciprocal insemination patterns per se does not prove trading (Anthes and Michiels 2005; Koene and Ter Maat 2005). In fact, reliable experimental evidence for such behaviours is still scarce, although an unequivocal example of conditional reciprocity (sperm trading in this case) was recently documented in the sea slug Chelidonura hirundinina using experimen- tally produced cheaters (Anthes et al. 2005). Thus, doubts persist about the occurrence of conditional reciprocity in many hermaphroditic species (Koene and Ter Maat 2005; Anthes et al. 2006a). We argue that one reason is that, so far, mostly pair experiments have been carried out. In such experiments, mating sessions are observed on two individuals only, which for instance does not allow for partner choice. Unless experimentally created cheaters are used, pair experiments showing non-random alternation of gender roles may be misleading. Patterns of alternation could stem from both individuals independently executing their own mating strategy and being enforced to do so with the same partner. Such frequent alternations resulting from unconditional reciprocity were recently documented in pairs of Lymnaea stagnalis individuals (Koene and Ter Maat 2005). In agreement with this observation, alternation of gender roles was never seen in spontaneous mating sequences observed in large groups of this species (ca. 700 snails per group, Koene and Ter Maat 2005). In addition, density (or group size) is expected to influence the evolution of mating behaviours such as conditional reciprocity. Recent models suggest that conditional reciprocity will be favoured when a gender conflict exists (i.e., one gender allows higher fitness gains than the other, so that individuals have a preference for this gender) and when mate-encounter rates are high so that retaliation against cheaters (i.e., preferring non-cheaters) is costless (see the "opportunistic male" and "gender ratio" hypotheses; Anthes et al. 2006a). In contrast, unconditional reciprocity would be favoured in low-conflict situations such as under balanced male and female benefits, but also under low mate-encounter rates when both defection and retaliation become costly (Puurtinen and Kaitala 2002; Anthes et al. 2006a). Investigating mating behaviours under contrasted densities should provide important additional insights into the proximate and ultimate determinants of reciprocity.

In this study, we will therefore examine mating behaviours under contrasted density levels using the species Physa acuta. This snail is a simultaneous and internally fertilizing hermaphroditic freshwater snail with a sperm-storage organ (Paraense and Pointier 2003). As in all Basommatopohran pulmonates, within one copulation, only one gender role can be performed at a time and reciprocity of gender role may follow (Wethington and Dillon 1996). Gender roles are thus clearly defined and can easily be distinguished. Non-random gender alternation over successive copulations has recently been observed in experimental pairs of individuals (Facon et al. 2007). However, it is not known at present whether this reciprocity is conditional or not.

Specifically, we will address the following questions: (1) Does conditional reciprocity in mating behaviour (i.e. gender-role preference) exist in this species? (2) Do snails adjust their mating behaviours to density? 


\section{Materials and methods}

Population studied and rearing protocol

Ten mature individuals $\left(G_{0}\right)$ were sampled in a small irrigation canal in Ecublens $\left(46^{\circ} 31^{\prime} \mathrm{N}, 6^{\circ} 32^{\prime} \mathrm{E}\right.$; Switzerland) that is often dry in summer and frozen in winter. They were brought back to the laboratory and isolated in 75-ml plastic boxes filled with water. As body size and age may influence the mating behaviour of $P$. acuta (Ohbayashi-Hodoki et al. 2004), we collected clutches only during the first 2 days in the lab to obtain $G_{1}$ offspring similar in age and size. Hence, size differences between individuals were too low to detect any effect of size on the tested variables (Results not shown). Two weeks later, $18 G_{1}$ offspring per $G_{0}$ individual (10 families) were each isolated in a 75-ml plastic box for five more weeks. After this period, individuals were allowed to mate freely as male and female, so as to avoid any bias due to reproductive history. To ensure this, $G_{1}$ juveniles from different families were mixed in boxes of ten individuals (one individual per family) for 2 weeks, after being marked with gouache paint (previously shown to be harmless; Henry et al. 2003). Subsequently, individuals were isolated again in $75-\mathrm{ml}$ plastic boxes for 2 weeks. Because of this isolation, all tested individuals should seek new copulations during the mating-behaviour observations. At the end of the rearing protocol, each of the $10 G_{0}$ families was composed of $18 G_{1}$ snails. Throughout the experiment, snails were maintained at $24^{\circ} \mathrm{C}$ under a $12 \mathrm{~L} / 12 \mathrm{D}$ photoperiod and fed ad libitum with boiled lettuce. Water was changed every 3 days.

\section{Mating behaviour observations and statistical analyses}

To experimentally manipulate population densities, three types of boxes were used: (1) a small box for high density (HD, $175 \times 115 \times 65 \mathrm{~mm}$ ), (2) a medium box for medium density (MD, $235 \times 175 \times 90 \mathrm{~mm}$ ) and (3) a large box for low density (LD, $335 \times 235 \times 130 \mathrm{~mm}$ ). Each box contained one randomly assigned individual per family (i.e., 10 individuals per box). Therefore, the simulated density

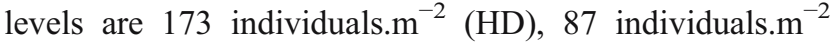
(MD), and 44 individuals. $\mathrm{m}^{-2}$ (LD), respectively. Six replicates per density level were set up. The mating behaviour of $P$. acuta snails was observed daily for $1 \mathrm{~h}$ in all boxes simultaneously over 6 consecutive days (at 2 p.m. everyday). In a previous experiment, it was shown that $1 \mathrm{~h}$ is sufficient for several copulations to take place between two individuals reared in conditions similar to the present study (up to seven copulations, Facon et al. 2006). A typical copulation involves (1) approach and climbing onto the shell of an intended female by a male-role snail, (2) crawling to the female gonophore, (3) preputium eversion and intromission (copulations may last up to $30 \mathrm{~min}$ ), and
(4) dismounting. Moreover, when acting as females, individuals can display rejection behaviours during a copulation such as shell swinging and phallus biting (Wethington and Dillon 1996; Ohbayashi-Hodoki et al. 2004; Facon et al. 2006). Thus, gender roles are easily distinguishable. We recorded the number of copulations per box, the identity (family) and gender role of every individual engaged in copulation. Statistical analyses were carried out using the software R (R Development Core team 2004). The effect of density on the number of copulations per box was tested through a repeated-measures ANOVA procedure, with time (in days) and density as main factors and box as an error term. This test was first made on the whole dataset, and then only on the first 2 days of the experiment. Comparisons between density levels were carried out using Tukey's Honest Significant Differences test.

The effect of density on gender alternation was tested by comparing the frequency of pairs of successive copulations involving the same individual with a change in gender roles among density treatments using Fisher's exact test. A binomial test was used for comparison of the proportion of cases where individuals switched gender role over two successive copulations. We tested whether this proportion differed from 0.5 (the proportion expected assuming that gender-role preference is random). Finally, the effect of density on the frequency of individuals that stayed with the same partner among those who alternated gender roles over two successive copulations was investigated using a Fisher's exact test. We then used a binomial test to compare the proportion of individuals staying with the same partner during gender alternation to the proportion expected assuming that assortment is random (i.e., 1/9; each individual has nine potential mates in any box).

\section{Results}

During the whole experiment, 149 individuals (out of 180 observed, $82.7 \%$ ) engaged in at least one copulation. In total, 163 copulations were observed. The number of copulations per individual per hour ranged from 0 to 3 with an average of $0.30 \pm 0.02$.

The variation in the number of copulations per box ( \pm standard errors) over time is shown in Fig. 1. A significant effect of time was found over the 6 days of the experiment (Table 1). No difference could be detected between LD, MD and HD boxes (Table 1) and the interaction between time and density was not significant (Table 1). Over the first 2 days, there was a significant effect of density on the number of copulations per box (Table 1) with HD boxes showing significantly more copulations than LD boxes (Tukey HSD, LD vs MD, $P=0.14$; LD vs HD, $P=0.007$; 
Fig. 1 Variation of the mean number of copulations per box with time (with standard errors). The bold line with triangles represents the high-density treatment. The large dashed line with squares represents the medium density treatment. The small dashed line with diamonds represents to the low-density treatment. Differences between treatments are only significant during the first 2 days of the experiment with high-density boxes showing more copulations than low-density boxes

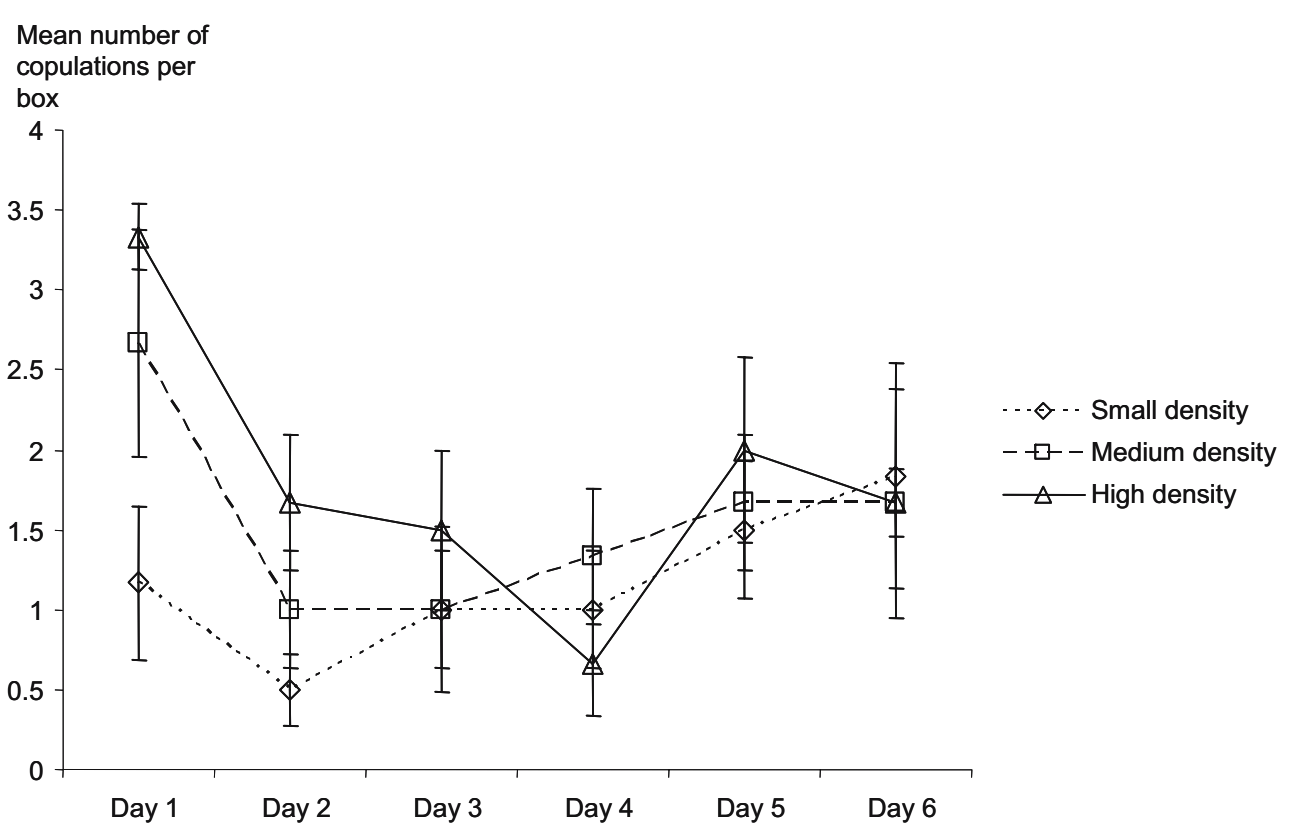

MD vs HD, $P=0.41)$. The number of copulations also decreased with time (Table 1) and the interaction between time and density was not significant (Table 1).

Density did not affect the frequency of gender-role alternation (Fisher's exact test, $N=53, P=0.66$ ). Among the 53 pairs of successive copulations, by the same individual, a switch in gender role occurred in 36 cases (67.9\%), which significantly departs from random gender choice (Fig. 2a, Binomial test, $P=0.01$ ). Thus, in $P$. acuta, gender switch occurs more often than expected by chance. Density did not affect the frequency of individuals staying with the same partner among those engaged in gender alternation (Fisher's exact test, $N=36, P=0.09)$. Among 36 individuals switching their gender role, only $3(8.3 \%)$ stayed with the same partner. This percentage does not depart from random partners assortment (Fig. 2b, Binomial test, $P=0.79$ ).
Therefore in $P$. acuta, individuals do not preferentially stay with the same partner when switching their gender role.

\section{Discussion}

In the present study, the basis for conditional reciprocity over gender roles and the possible adjustments of mating behaviours to density were investigated in the simultaneous hermaphrodite Physa acuta using individually marked snails reared at three contrasted density levels. The simulated density levels were shown to affect the frequency of copulations during the first 2 days after isolation, with significantly more copulations in high-density groups than in low-density groups. This suggests that the low-density treatment was sufficient to limit mate-encounter probabilities.

Table 1 Results of the repeated-measures ANOVA procedure testing the effects of density and time on the number of copulations per box

\begin{tabular}{|c|c|c|c|c|c|}
\hline & Factor & $D f$ & SS & $F$ value & $P$ value \\
\hline \multicolumn{6}{|l|}{ Over 6 days } \\
\hline \multirow[t]{2}{*}{ Error: box } & Density & 2 & 7.46 & 2.36 & 0.13 \\
\hline & Residuals & 15 & 23.69 & & \\
\hline \multirow[t]{3}{*}{ Error: within } & Time & 5 & 26.05 & 4.11 & 0.002 \\
\hline & Density/time & 10 & 14.65 & 1.15 & 0.33 \\
\hline & Residuals & 75 & 95.14 & & \\
\hline \multicolumn{6}{|c|}{ Over the first 2 days } \\
\hline \multirow[t]{2}{*}{ Error: box } & Density & 2 & 16.89 & 6.23 & 0.01 \\
\hline & Residuals & 15 & 20.33 & & \\
\hline \multirow[t]{3}{*}{ Error: within } & Time & 1 & 16.00 & 17.14 & 0.0009 \\
\hline & Density/time & 2 & 2.00 & 1.07 & 0.37 \\
\hline & Residuals & 15 & 14.00 & & \\
\hline
\end{tabular}

The test was first made on the whole dataset (over 6 days), and then only on the first 2 days of the experiment 


\section{Number of pairs of successive copulations}

a
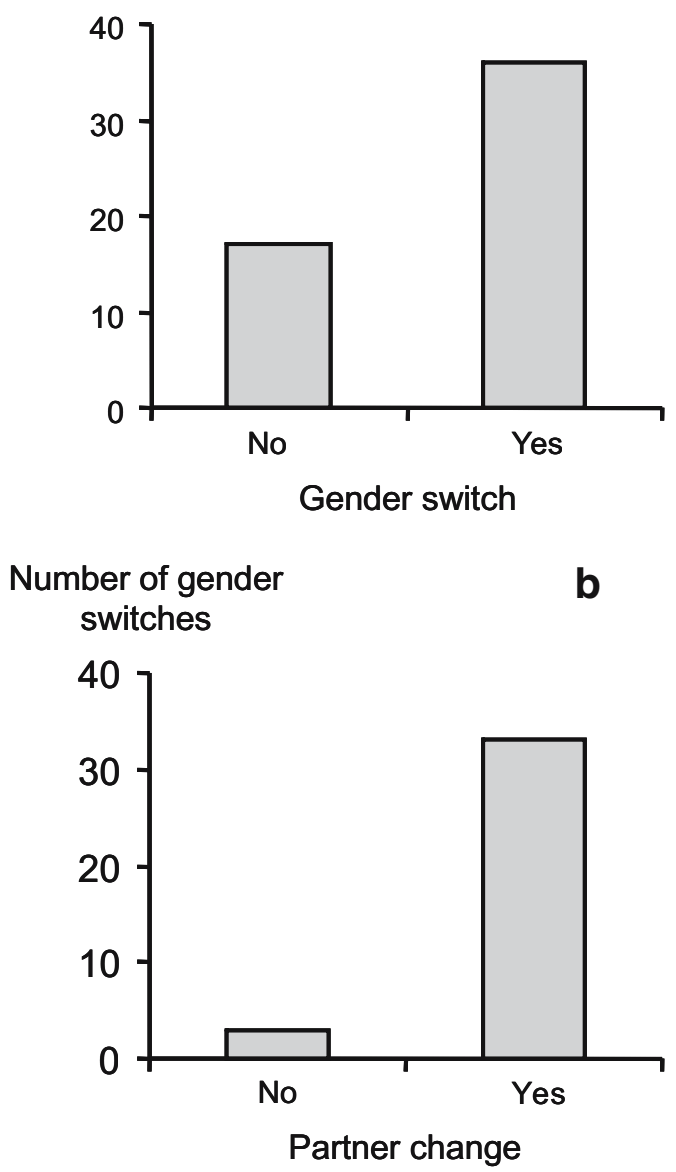

Fig. 2 Physa acuta snails do alternate gender roles but not preferentially with the same partner. a Numbers of pairs of successive copulations by the same individual with and without gender switch observed over the whole experiment. These proportions significantly deviate from random gender choice. b Numbers of gender switches with and without partner change observed over the whole experiment. These proportions do not significantly deviate from random partner assortment

If mate-searching behaviours (e.g., using diluted chemical cues or mucus trails) exist in this species, they are not efficient enough to counteract differences of mate-encounter rate between the densities used here. Furthermore, previous studies based on pair observation have found no correlation between the time of the first contact between partners and other variables such as partner relatedness, shell size or mating history (Wethington and Dillon 1996; Facon et al. 2006; Facon et al. 2007). Therefore, mate encounters are likely determined by the random movement of individuals in their environment. After 2 days of group living, copulation rates no longer differed between density treatments, mainly because of a decrease in high- and medium-density treatments. This result suggests that the limitation imposed by low density on mate-encounter rates is rapidly counterbalanced by a decrease in eagerness associated with either depleted autosperm stores or the huge capacity of allosperm storage of this species (Wethington and Dillon 1991; Dillon et al. 2005). In agreement with this hypothesis, it has been shown that outcrossing rates in the field are very high and not limited by density $(t>0.9$ even in small ponds or temporary habitats; Henry et al. 2005).

Our results revealed that these snails alternate gender roles over successive copulations more often than expected by chance independently of density level. In agreement with this pattern, high gender-alternation rates were found in a previous study based on the observation of pairs of snails isolated for short or long periods of time (Facon et al. 2007). Thus, it seems that gender-role alternation rate is high independently of population density in this species.

When performing two successive copulations with genderrole alternation, snails did not preferentially stay with the same partner. Note that this result is robust to the assumption that all individuals were willing to mate during the experiment. Indeed, that some individuals might not be available as a partner (for any reason such as illness) would only increase the probability to mate twice with the same partner under random mating. We find that this probability is slightly lower than expected under random mating (3/36 vs $1 / 9)$. Our test is thus conservative. Not only the rate of individuals that are not available as a partner must be low, but also individuals do not particularly seek for copulations with a partner they already copulated with. We were unable to test whether there was an effect of the first gender role performed on the propensity to switch partners in a subsequent copulation due to insufficient statistical power. Again, we found no effect of density on the propensity to switch partners. However, the significance of this result is weak. In particular, we cannot firmly establish that the proportion of gender-role swapping between mating partners (i.e., reciprocation) is not higher at low density than at medium and high densities because too few (6) observations of gender-role switching were available in the low-density treatment. Furthermore, the range of densities used in this experiment, which was much narrower than in the field $\left(<1\right.$ individual. $\mathrm{m}^{-2}$ to several hundreds individuals. $\mathrm{m}^{-2}$, Henry et al. 2005; B.F. personal observation), may have limited our ability to detect an effect of density on gender-role alternation. Confirming this hypothesis that, even at very low densities, snails do not engage in conditional reciprocity may be very difficult in practice unless techniques for experimentally producing cheaters are developed (as exemplified in Anthes and Michiels 2005). However, according to our results, conditional reciprocity does not rule decisions over gender roles in moderate-density populations of $P$. acuta. Instead, it seems that gender-role alternation results from each individual independently executing its own mating strategy.

A key prerequisite for conditional reciprocity to evolve is that conflict over gender role should be common, i.e., most individuals in this species should share a preference 
for the same gender role (Leonard 2005). In P. acuta, there is growing evidence that gender-role preference may vary within the life of an individual, e.g., with individual size (and thus age; Ohbayashi-Hodoki et al. 2004) and reproductive history (Wethington and Dillon 1996; Anthes et al. 2006b; Facon et al. 2007). Hence, at any given time, not all individuals should share the same gender-role preference, which may strongly attenuate conflicts over gender role. In P. acuta, it was notably shown that snails isolated for a short period of time (2 to 6 days) prefer copulating as males and that the probability of accepting a copulation as a female increases with the time spent alone (Wethington and Dillon 1996; Facon et al. 2007). An interpretation may be that gender-role preference in this species is driven by the relative amounts of autosperm and allosperm available in an individual snail's reproductive tract. According to this hypothesis, after male copulation, snails should suffer from autosperm depletion and thus either stop seeking for copulation or prefer the female role until their store of autosperm is replenished. In contrast, after female copulation, snails should have sufficient allosperm stores to enable high-quality egg fertilisation. They should thus seek for opportunities to increase sperm donation. Gender-role alternation in P. acuta would thus follow the dynamics of individual allosperm and autosperm stores rather than the rule of conditional reciprocity. Congruent with this hypothesis, a recent pair experiment on gender-role alternation found that, independent of isolation status (and thus genderrole preference), partners alternated gender role equally (Facon et al. 2007). Gender-role alternation would thus not be an adaptive response to the intensity of gender conflict in this species (Facon et al. 2007).

\section{Occurrence of conditional reciprocity}

Conditional reciprocity used to be considered widespread among internally fertilising hermaphrodites (e.g., Leonard 1990). Although conditional reciprocity is a theoretically appealing idea, direct and convincing evidence of its existence in internally fertilizing hermaphrodites is still scarce (Chaine and Angeloni 2005; Koene and Ter Maat 2005; Anthes et al. 2006a). There is growing suspicion that, in a number of studies, patterns compatible with conditional reciprocity may actually stem from unconditional reciprocity. This has notably been clearly established for patterns of gender-role alternation in the freshwater snail Lymnaea stagnalis (Koene and Ter Maat 2005), and correlation in partner behaviours (none of the partners mate as sperm donor or both mate as sperm donors) in the opisthobranch Bulla gouldiana (Chaine and Angeloni 2005). The present study supports the recent advocation of several authors (Chaine and Angeloni 2005; Koene and Ter Maat 2005; Anthes et al. 2006a) that pair experiments with non- manipulated individuals may be misleading about conditional reciprocity. In addition, the widely held view of a species-wide constant gender-role preference leading to frequent gender-role conflict is increasingly questioned by recent empirical studies. As predicted by theory (Angeloni et al. 2002), gender preferences vary during the life of an individual in relation to body size in a number of species of freshwater snails (Physa sp.; DeWitt 1991; OhbayashiHodoki et al. 2004), opisthobranchs (e.g., Angeloni and Bradbury 1999; Angeloni 2003; Gianguzza et al. 2004; Chaine and Angeloni 2005) and cestodes (Lüscher and Wedekind 2002). Partner availability has been shown to generate biases in gender-role allocation (Trouvé et al. 1999; Tan et al. 2004; Schärer et al. 2005; Schleicherova et al. 2006). There is also growing evidence that reproductive history (or time since the last copulation) may influence gender-role preference (Van Duivenboden and Ter Maat 1985; Wethington and Dillon 1996; De Boer et al. 1997; Michiels and Streng 1998; Michiels and Bakovski 2000; Koene and Ter Maat 2005; Facon et al. 2007). Gender-role preference was even shown to change within a mating sequence (in the sea slug Navanax inermis; Michiels et al. 2003). Thus, it is possible that conflict over the desired gender role (and thus selection favouring conditional reciprocity) is far less common than previously thought (e.g., Chase and Vaga 2006). Finally, theoretical developments suggest that conditional reciprocity is not expected to be particularly stable under any specific ecological conditions (reviewed in Doebeli and Hauert 2005). For instance, very low and very high mate-encounter rates may favour unconditional reciprocity and "hit-and-run" strategies, respectively (Anthes et al. 2006a). Therefore, even within a species, the propensity for conditional reciprocity might vary among individuals and/or populations and be adjusted to environmental cues.

All this clearly advocates for more work on internally fertilizing hermaphrodites. First, experiments using experimentally produced cheaters, group experiments and field observations are definitely needed to assess the actual frequency of conditional reciprocity in hermaphrodites (Anthes et al. 2006a). Second, at present, conditional reciprocity has only been unambiguously established in the sea slug Chelidonura hirundinina (Anthes et al. 2005). It has also been proposed in a few other species based on convincing pieces of evidence such as correlated copulation durations (e.g., Navanax inermis, Leonard and Lukowiak 1984, 1991; Michiels et al. 2003), correlated amounts of sperm transferred and received (e.g., Succinea putris, Jordaens et al. 2005) or avoidance of already mated partners (e.g., Aeolidiella glauca, Karlsson and Haase 2002). A common feature of all these species is that they are able to simultaneously transfer sperm. Hence, no species where only one gender role can be performed at a 
time has been recognized as a good candidate for conditional reciprocity. In such species, the partner that first performs the non-preferred gender role cannot ensure that it will be given an opportunity to play the preferred role in the next copulation. The risk of being cheated on is therefore likely to be higher than in species where insemination is simultaneous. As well-documented case studies will accumulate, it will be interesting to see whether the potential for simultaneous inseminations is an important constraint on the evolution of conditional reciprocity. Finally, our observation that gender preference is not fixed and similar for every individual in a given species calls for models of the evolution and maintenance of hermaphroditism that integrate variation of gender-role preference on one hand and models with more explicit ecological settings (such as space and population density) on the other.

Acknowledgement BF benefited from a Lavoisier post-doctoral grant from the Ministère Français des Affaires Etrangères. This study was supported by grant $31-108194 / 1$ from the Swiss National Foundation. We are grateful to C. Benetollo, E. Chapuis, and L. Sauteur for their help in snail rearing as well as P. Jarne and J. Auld for fruitful comments on the manuscript. This research complies with current laws in Switzerland. This is contribution number 2007-088 of ISEM.

\section{References}

Angeloni L (2003) Sexual selection in a simultaneous hermaphrodite with hypodermic insemination: body size, allocation to sexual roles and paternity. Anim Behav 66:417-426

Angeloni L, Bradbury J (1999) Body size influences mating strategies in a simultaneously hermaphroditic sea slug, Aphysia vaccaria. Ethol Ecol Evol 11:187-195

Angeloni L, Bradbury JW, Charnov EL (2002) Body size and sex allocation in simultaneously hermaphroditic animals. Behav Ecol 13:419-426

Anthes N, Michiels NK (2005) Do "sperm trading" simultaneous hermaphrodites always trade sperm? Behav Ecol 16:188-195

Anthes N, Putz A, Michiels NK (2005) Gender trading in a hermaphrodite. Curr Biol 15:R792-R793

Anthes N, Putz A, Michiels NK (2006a) Sex role preferences, gender conflict and sperm trading in simultaneous hermaphrodites: a new framework. Anim Behav 72:1-12

Anthes N, Putz A, Michiels NK (2006b) Hermaphrodite sex role preferences: the role of partner body size, mating history and female fitness in the sea slug Chelidonura sandrana. Behav Ecol Sociobiol 60:359-367

Axelrod R, Hamilton WD (1981) The evolution of cooperation. Science 211:1390-1396

Bateman AJ (1948) Intra-sexual selection in Drosophila. Heredity 2:349-368

Bishop JDD, Pemberton AJ (2006) The third way: spermcast mating in sessile marine invertebrates. Integr Comp Biol 46:398-406

Chaine A, Angeloni L (2005) Size-dependent mating and gender choice in a simultaneous hermaphrodite, Bulla gouldiana. Behav Ecol Sociobiol 59:58-68
Charnov EL (1979) Simultaneous hermaphroditism and sexual selection. Proc Natl Acad Sci USA 76:2480-2484

Chase R, Vaga K (2006) Independence, not conflict, characterizes dart-shooting and sperm exchange in a hermaphroditic snail. Behav Ecol Sociobiol 59:732-739

Dall SRX, Wedell N (2005) Evolutionary conflict: sperm wars, phantom inseminations. Curr Biol 15:R801-R803

De Boer PACM, Jansen RF, Koene JM, Ter Maat A (1997) Nervous control of male sexual drive in the hermaphroditic snail Lymnaea stagnalis. J Exp Biol 200:941-951

DeWitt TJ (1991) Mating behavior of the freshwater pulmonate snail, Physa gyrina. Am Malacol Bull 9:81-84

Dillon RT, McCullough TE, Earnhardt CE (2005) Estimates of natural allosperm storage capacity and self-fertilization rate in the hermaphroditic freshwater pulmonate snail, Physa acuta. Invert Repr Dev 47:111-115

Doebeli M, Hauert C (2005) Models of cooperation based on the Prisoner's Dilemma and the Snowdrift game. Ecol Lett 8:748-766

Facon B, Ravigné V, Goudet J (2006) Experimental evidence of inbreeding avoidance in the hermaphroditic snail Physa acuta. Evol Ecol 20:395-406

Facon B, Ravigné V, Sauteur L, Goudet J (2007) Effect of mating history on gender preference in the hermaphroditic snail Physa acuta. Anim Behav (in press)

Fischer EA (1980) The relationship between mating system and simultaneous hermaphroditism in the coral reef fish, Hypoplectrus nigricans (Serranidae). Anim Behav 28:620-633

Gianguzza P, Badalamenti F, Jensen KR, Chemello R, Cannicci S, Riggio S (2004) Body size and mating strategies in the simultaneous hermaphrodite Oxynoe olivacea (Mollusca, Opistobranchia, Sacoglossa). Funct Ecol 18:899-906

Henry PY, Pradel R, Jarne P (2003) Environment-dependent inbreeding depression in a hermaphroditic freshwater snail. J Evol Biol 16:1211-1222

Henry PY, Bousset L, Sourrouille P, Jarne P (2005) Partial selfing, ecological disturbance and reproductive assurance in an invasive freshwater snail. Heredity 95:428-436

Jordaens K, Pinceel J, Backeljau T (2005) Mate choice in the hermaphroditic land snail Succinea putris (Stylommatophora: Succineidae). Anim Behav 70:329-337

Karlsson A, Haase M (2002) The enigmatic behaviour and reproduction of a simultaneous hermaphrodite, the nudibranch Aeolidiella glauca (Gastropoda, Opisthobranchia). Can J Zool 80:260-270

Koene JM, Ter Maat A (2005) Sex role alternation in the simultaneously hermaphroditic pond snail Lymnaea stagnalis is determined by the availability of seminal fluid. Anim Behav 69:845-850

Leonard JL (1990) The hermaphrodite's dilemma. J Theor Biol 147:361-372

Leonard JL (1999) Modern portfolio: theory and the prudent hermaphrodite. Invert Repr Dev 36:129-135

Leonard JL (2005) Bateman's principle and simultaneous hermaphrodites: a paradox. Integr Comp Biol 45:856-873

Leonard JL (2006) Sexual selection: lessons from hermaphrodite mating systems. Integr Comp Biol 46:349-367

Leonard JL, Lukowiak K (1984) Male-female conflict in a simultaneous hermaphrodite resolved by sperm trading. Am Nat 124:282-286

Leonard JL, Lukowiak K (1991) Sex and the simultaneous hermaphrodite: testing models of male-female conflict in a sea slug, Navanax inermis (Opistobranchia). Anim Behav 41:255-266

Locher R, Baur B (2000) Mating frequency and resource allocation to male and female function in the simultaneous hermaphrodite land snail Arianta arbustorum. J Evol Biol 13:607-614

Lüscher A, Wedekind C (2002) Size-dependent discrimination of mating partners in the simultaneous hermaphroditic cestode Schistocephalus solidus. Behav Ecol 13:254-259 
Michiels NK (1998) Mating conflicts and sperm competition in simultaneous hermaphrodites. In: Birkhead TR, Moller AP (eds) Sperm competition and sexual selection. Academic, London, pp 219-254

Michiels NK, Bakovski B (2000) Sperm trading in a hermaphroditic flatworm: reluctant fathers and sexy mothers. Anim Behav 59:319-325

Michiels NK, Streng A (1998) Sperm exchange in a simultaneous hermaphrodite. Behav Ecol Sociobiol 42:171-178

Michiels NK, Raven-Yoo-Heufes A, Kleine Brockmann K (2003) Sperm trading and sex roles in the hermaphroditic opistobranch sea slug Navanax inermis: eager females or opportunistic males? Biol J Linn S 78:105-116

Ohbayashi-Hodoki K, Ishihama F, Shimada M (2004) Body sizedependent gender role in a simultaneous hermaphrodite freshwater snail, Physa acuta. Behav Ecol 15:976-981

Paraense WL, Pointier JP (2003) Physa acuta draparnaud,1805 (Gastropoda: Physidae): a study of topotypic specimens. Mem Inst Oswaldo Cruz 98:513-517

Puurtinen M, Kaitala V (2002) Mate-search efficiency can determine the evolution of separate sexes and the stability of hermaphroditism in animals. Am Nat 160:645-660

R Development Core Team (2004) R: a language and environment for statistical computing. R Foundation for Statistical Computing, Vienna, Austria. URL: http://www.R-project.org. ISBN3900051-00-3
Schärer L, Sandner P, Michiels NK (2005) Trade-off between male and female allocation in the simultaneously hermaphroditic flatworm Macrostomum sp. J Evol Biol 18:396-404

Schleicherova D, Lorenzi MC, Sella G (2006) How outcrossing hermaphrodites sense the presence of conspecifics and suppress female allocation. Behav Ecol 17:1-5

Tan GN, Govedich FR, Burd M (2004) Social group size, potential sperm competition and reproductive investment in a hermaphroditic leech, Helobdella papillomata (Euhirudinea: Glossiphoniidae). J Evol Biol 17:574-580

Trouvé S, Jourdane J, Renaud F, Durand P, Morand S (1999) Adaptive sex allocation in a simultaneous hermaphrodite. Evolution 53:1599-1604

Van Duivenboden YA, Ter Maat A (1985) Masculinity and receptivity in the hermaphrodite pond snail, Lymnaea stagnalis. Anim Behav 33:885-891

Vreys C, Michiels NK (1998) Sperm trading by volume in a hermaphroditic flatworm with mutual penis intromission. Anim Behav 56:777-785

Wethington AR, Dillon RT (1991) Sperm storage and evidence for multiple inseminations in a natural population of the freshwater snail, Physa. Am Malacol Bull 9:99-102

Wethington AR, Dillon RT (1996) Gender choice and gender conflict in a non-reciprocally mating simultaneous hermaphrodite, the freshwater snail, Physa. Anim Behav 51:1107-1118 